\title{
Earthquake Waves Reflected at the Inside of the Core Boundary*
}

\author{
B. Gutenberg \\ Seismological Laboratory \\ California Institute of Technology \\ Pasadena, California
}

\begin{abstract}
Travel times of waves generated by an earthquake at a depth of $600 \mathrm{~km}$ and reflected from the inside of the core boundary as well as the epicentral distances of the caustics of PKKP, SKKP, and PKKS agree within the limits of error with those calculated on the basis of velocity-depth curves. This applies also to the travel times of SKKS and SKKKS for which poor agreement was reported before the inner core was established. The periods of all waves traveling through the outer core seem to be shortened. In the outer core the attenuation is possibly smaller than in the mantle.
\end{abstract}

Symbols and materials used-For brevity in the following discussion, waves which have traveled through the outer core, but not through the inner core, are indicated by one prime, those which have entered the inner core, by two primes. Thus, for example, $\mathbf{P}^{\prime} \equiv \mathbf{P K P}$, $\mathrm{P}^{\prime \prime} \equiv$ PKIKP, SKKP" $\equiv$ SKIKKIKP. Symbols with a subscript 1 (for example, $P_{1}{ }^{\prime}$ ) refer to the earlier of two branches connecting at a focal point, those with a subscript 2 to the later. An asterisk indicates an epicentral distance over $180^{\circ}$ (for example, SKKS*).

Unless it is stated otherwise, all data refer to the earthquake of 1957, April 16, 4:04:04, with an adopted epicenter at $4.5^{\circ} \mathrm{S}, 107.5^{\circ} \mathrm{E}$, focal depth $600 \mathrm{~km}$. The elements of this shock have been redetermined. Considering the expected systematic errors involved in the calculation (for example, in the assumed travel times or resulting from the assumption that the internal structure of the earth is spherical), the coordinates of the adopted epicenter are probably correct within a few tenths of a degree, the origin time within 2 or 3 seconds, and the focal depth within about $20 \mathrm{~km}$. Records of this shock from 55 stations at distances from $119^{\circ}$ to $178^{\circ}$ have been used previously by Gutenberg [1958a, p. 244]. Additional records have been made available upon request by the following stations (epicentral distance in degrees in parentheses): Brisbane

\footnotetext{
* Contribution No. 933, Division of Geological Sciences, California Institute of Technology.
}

(48.9), Matsushiro (49.9), Jerusalem (77.4), Kiruna (93.0), Uppsala (93.8), Honolulu (95.7), Skalstugan (96.3), College (100.3), Sitka (108.1), Alberni (117.3), Victoria (118.5), Banff (121.3), Saskatoon (124.8). The author is very grateful for the speedy response from all stations.

Theoretical travel times of waves originating at the earth's surface have been calculated on the basis of the tables of Jeffreys and Bullen [1940] for waves through the mantle. For those through the core the same procedure has been followed as described by Gutenberg [1958a, p. 241]. This is based on the travel times of Gutenberg [1958b, Table 4] for the portion of the waves through the core and on the travel times of Jeffreys and Bullen [1940] for $\mathrm{PcP}, \mathrm{ScS}$, and ScP for zero focal depth. Corrections for the focal depth of $600 \mathrm{~km}$ have been taken either from tables of Jeffreys and Bullen [1940] or from tables of Gutenberg and Richter [1936]. The resulting differences between the two procedures are small and within the limits of errors. Corrections for the effect of the focal depth on distances of the focal points have been taken from tables of Gutenberg and Richter [1936]. Errors in the assumption of corresponding portions of $\mathrm{PcP}$, ScS, and PcS on the one hand and of the portions of the paths inside the core on the other rarely affect the resulting travel-time curves by more than 2 seconds; moreover, they may shift the calculated distance of focal points by 


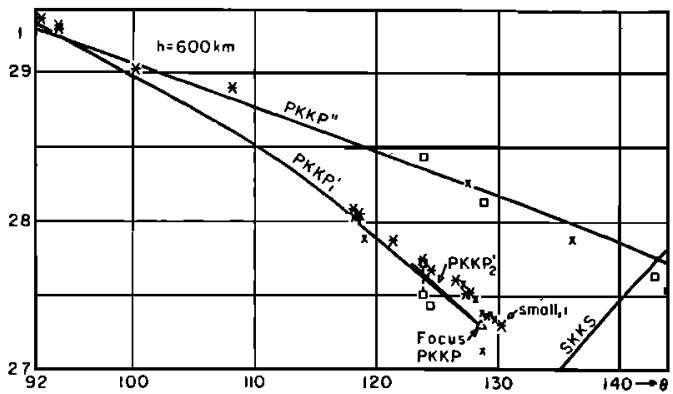

FIg. 1-Travel times $t$ of the PKKP group for epicentral distances $\theta$ in degrees, observed for earthquake 1957, April 16, at 4:04:04, focal depth $h=600 \mathrm{~km}$, and calculated curves for $h=600$ km. Symbols as in Fig. 3.

about one degree and even more under unfavorable circumstances; for example, for repeated reflections. The calculated travel-time curve then either extends beyond the actual curve in the preceding direction or ends at a shorter distance with a focal point practically on the actual curve.

The PKKP group-Travel times and amplitudes of PKKP' and PKKP" have been studied previously by the author [Gutenberg, 1951, pp. 381, 383; Gutenberg, 1958a, p. 246]. Most of the travel times of $\mathrm{PKKP}^{\prime}$ observed for the shock $600 \mathrm{~km}$ deep are within a few seconds of the calculated values (Fig. 1). There are a few observations which closely fit the travel times calculated for PKKP", but this phase is never conspicuous. Some of the PKKP' phases, however, are very clear (Fig. 2). According to Jeffreys and Bullen [1940, p. 42] the focus of

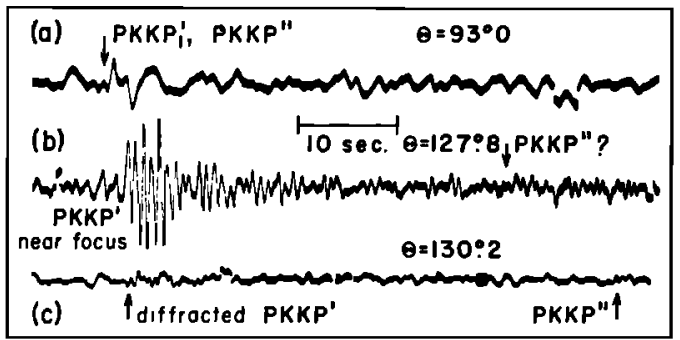

FIG. 2-Portions of records of shock 1957, April 16, 4:04:04, focal depth $600 \mathrm{~km}$, showing waves of the PKKP group. (a) Grenet vertical, Kiruna, $93.0^{\circ}$; (b) and (c) short-period Benioff verticals; (b) Woody, $127.8^{\circ}$; (c) Barrett, $130.2^{\circ}$, about 21/2 beyond focus of PKKP'.
PKKP' for shocks at zero focal depth should be at $234^{\circ}\left(126^{\circ}\right)$, whereas the new calculations give about $127^{\circ}$. The decrease in distance connected with a focal depth of $600 \mathrm{~km}$ is about $2^{\circ}$, so that theoretically the caustic of PKKP' should be expected at $232^{\circ}$ to $231^{\circ}$; that is, near $128^{\circ}$ to $129^{\circ}$. Actually, no PKKP' waves have been observed beyond $130.2^{\circ}$ (Fig. 2c), although there are records available for $130.7^{\circ}$, $132.2^{\circ}, 133.9^{\circ}, 134.2^{\circ}, 134.7^{\circ}$, etc.

The ratio of the amplitude $a$ to the period $T$ of PKKP' has been compared with the corresponding ratios for PP and for $\mathrm{P}^{\prime \prime}$. Unfortunately, the travel-time curve of $\mathrm{PP}$ crosses that for $\mathrm{pP}^{\prime \prime}$ near $130^{\circ}$ and follows it at greater distances. Consequently, amplitudes of $\mathrm{PP}$ cannot be found with confidence for distances exceeding about $1281^{\circ}{ }^{\circ}$. For distances less than $128^{\circ}$, the ratio $a / T$ for $\mathrm{PKKP}$ to that for $\mathrm{P}^{\prime \prime}$ was found to increase on seismograms for the short-period vertical instruments from about 0.1 to 0.2 at distance between $121^{\circ}$ and $127^{\circ}$ to 0.4 at $127.5^{\circ}$ and 0.5 at $127.8^{\circ}$ (Fig. 2b) and then to drop to less than 0.1 beyond $128^{\circ}$ (Fig. 2c). Similarly, the ratio of $a / T$ for $\mathrm{PKKP}^{\prime}$ to that for PP was 1 or more at distances less than $128^{\circ}$ but only about 0.5 between $128^{\circ}$ and $12812^{\circ}$. Thus the focal point of PKKP' in the shock $600 \mathrm{~km}$ deep has been at about $12734^{\circ}$, and diffracted PKKP waves have been observed for about $2 \frac{1}{2}{ }^{\circ}$ beyond. Thus, the travel-time curve for PKKP, calculated on the recent assumptions of the velocities of longitudinal waves in the core, agrees with the observations well within the limits of error.

PKKKP should have a caustic at distances near $315^{\circ}\left(45^{\circ}\right)$ with travel times near 36 minutes in shallow shocks (near 35 minutes in shocks $600 \mathrm{~km}$ deep), with both branches extending towards the epicenter. Apparently it has not been observed; in the present research no records obtained at such short distances have been used.

SKKS and SKKKS-Gutenberg and Richter [1939, p. 126] found noticeable differences between the observed and calculated travel times of SKKS and SKKKS; however, their travel times were calculated prior to the discovery of the inner core. A comparison of observed and calculated travel times of these phases was made by Nelson [1954], who found that the 


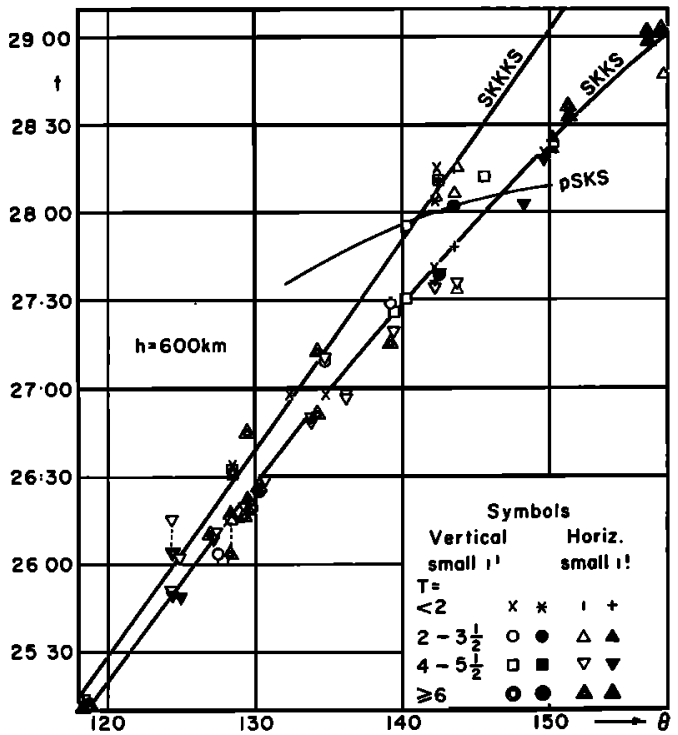

FIa. 3-Travel times $t$ of SKKS and SKKKS, details as in Fig. 1.

residuals for SKKS and SKKKS never exceeded 7 seconds, relative to the improved travel-time curves. Figure 3 shows a portion of the recently calculated travel times of the two phases for shocks at a focal depth of 600 $\mathrm{km}$ and the times observed on seismograms of the shock of April 16, 1957. The agreement is good. On seismograms recorded near a distance of $180^{\circ}$, where both phases should have a focal point, SKKS, SKKS*, SKKKS, and

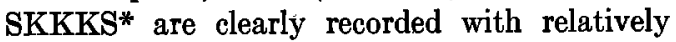
large amplitudes. At distances beyond $180^{\circ}$ there are some observations near the calculated curves for SKKS ${ }^{*}$ and SKKKS ${ }^{\prime *}$, and also a few near the calculated curve for SKKS ${ }^{\prime *}$, but in several instances these observed waves may belong to different phases with travel-time curves crossing that of the phase under consideration. SKKKS ${ }^{\prime \prime *}$ should start near a distance of $60^{\circ}$ with a travel time of about $431 / 2$ $\min$ for $h=600 \mathrm{~km}$, and its travel-time curve should continue across the epicenter where its travel time is about 45 minutes.

It is surprising how relatively large the amplitudes of SKKS and SKKKS are (compare Fig. 4 with Figs. 2 and 6). Nelson [1954, p. 46] found that in shallow shocks the observed amplitudes of the vertical component of SKKS'

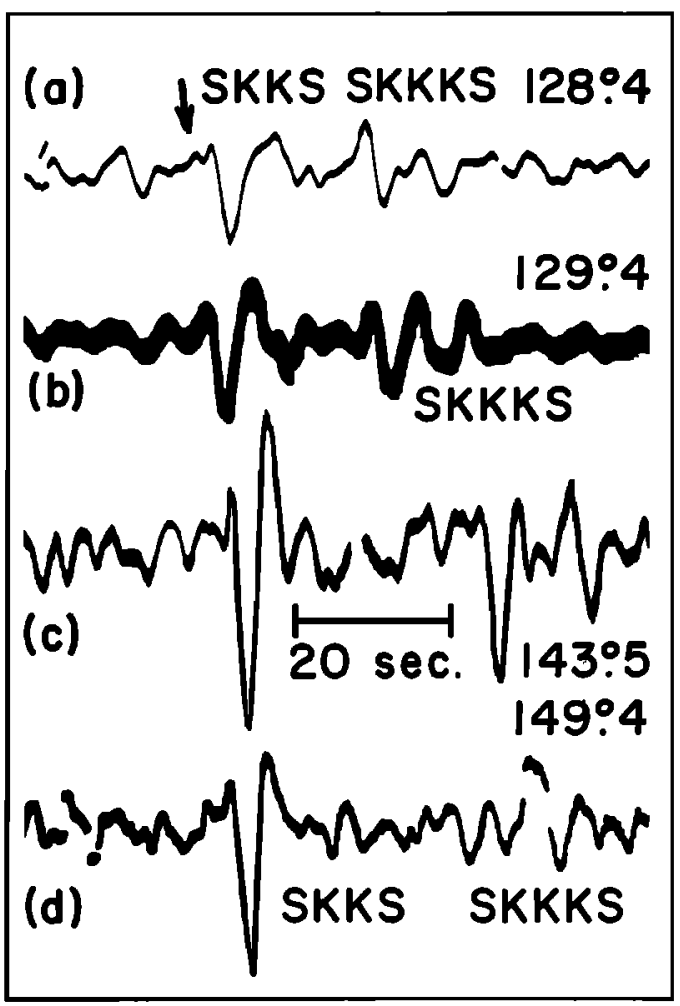

FIG. 4-Portions of records as in Fig. 2, showing SKKS and SKKKS. (a) long-period Benioff NS, Pasadena, $128.4^{\circ}$; (b) McComb-Romberg EW, Salt Lake City, $129.4^{\circ}$; (c) and (d) Wilson-Lamison NS; (c) Fayetteville, $143.5^{\circ}$; (d) Columbia, $149.6^{\circ}$.

at distances between $90^{\circ}$ and $135^{\circ}$ are between $11 / 2$ and 3 times the calculated amplitudes. The amplitudes of the horizontal components are close to the calculated values near $95^{\circ}$ and between $140^{\circ}$ and $150^{\circ}$, but are otherwise too small up to $170^{\circ}$ and only about one third of the calculated amplitudes near $120^{\circ}$ and $170^{\circ}$. Nelson [1954, p. 48] found an appreciable effect of prevailing directions of faulting for different locations of the sources. The remaining discrepancies at least partly may be the result of errors in the quantities assumed for the calculation of the observed amplitudes as well as for those found theoretically. Major errors in theoretical calculations may result from the fact that the periods $T$ of SKKS and SKKKS are between 1 and more than $10 \mathrm{sec}-$ onds, so that the corresponding lengths of the 
waves which arrive at the surface of the earth are between a few and more than $30 \mathrm{~km}$. The longer wavelengths are of the order of the thickness of the earth's crust. Consequently, the angle of incidence $i_{0}$ at the surface to be used for calculations, especially for the ratio of the ground displacement to the incident amplitude, may vary considerably as a function of $T$. Moreover, the ratio of the observed horizontal component $H$ to that of the vertical component $Z$ depends considerably on the angle of incidence $i_{0}$; in a homogeneous material this ratio may vary from about 5 for an angle $i_{0}$ near $10^{\circ}$ (short waves) to $2 \frac{1}{2}$ for an angle of $17^{\circ}$, which has to be assumed for relatively long waves as a first approximation. There are other sources of error in the theoretical calculations.

The fact that SKKS is rather large as compared with PKKP depends partly on the percentage of energy reflected at the core boundary (KK). For PKKP, the ratio of the amplitude of the reflected $K$ wave to that of the incident $\mathrm{K}$ wave should rarely if ever be greater than 0.1 [Gutenberg, 1951, p. 387]. On the other hand, in SKKS (and similarly in SKKKS) this ratio is 0.8 or more, if the angle of incidence $i$ inside the core boundary is between about $35^{\circ}$ (critical angle for $\mathbf{P}$ waves refracted into the mantle) and about $70^{\circ}$ [Dana, 1944, p. 194]; these conditions are fulfilled for SKKS at epicentral distances up to about $180^{\circ}$ and for SKKKS up to about $250^{\circ}$ (all observed SKKKS waves and SKKKS* have distances between about $180^{\circ}$ and $110^{\circ}$ ). Theoretically the factor $F$ for the decrease in amplitudes at all refractions and reflections at the core boundary is less than about 0.08 for practically all PKKP waves, near 0.5 for SKKS and near 0.4 for SKKKS as well as for SKKKS* at distances over $100^{\circ}$, but much smaller for SKKS*.

The relatively large amplitudes of SKKS and SKKKS indicate that the attenuation in the outer core is relatively small. Unfortunately, the travel-time curve for SKKKS* intersects so many travel-time curves of other phases that it is difficult to decide how much these phases contribute to the observed amplitudes attrributed to SKKKS*. On the other hand, the ratio of the amplitudes of SKKKS to those of SKKS or of SKS varies too much for a reliable calculation to be made of the absorption coefficient in the outer core. However, most calculated values for the absorption coefficient in the outer core for the shock of April 16,1957 , indicate that its value is smaller than in the mantle. If the absorption coefficients were equal in the mantle and outer core, SKKKS* should be about 0.5 of SKKS at $150^{\circ}$, if both have the same periods.

$P K K S, S K K P$ and related phases-Travel times of waves belonging to this group have been calculated, but little research has been done to find the relationship of the observed to the calculated times. "There is a long train of these waves with an indefinite beginning, so that the readings scatter very much when plotted, and do not serve to define a travel time curve" [Gutenberg and Richter, 1934, p. 118]. Figure 5, which shows a portion of the travel-time curves of this group but does not contain waves of the type pPKKS, etc., explains the long duration and the complicated appearance of this group (Fig. 6). Moreover, these waves have the same small ratio of the reflected to the incident amplitudes at the core boundary (KK) as PKKP, and the amplitude loss factor $F$ for refractions and reflections at the core boundary is even slightly smaller than that for PKKP.

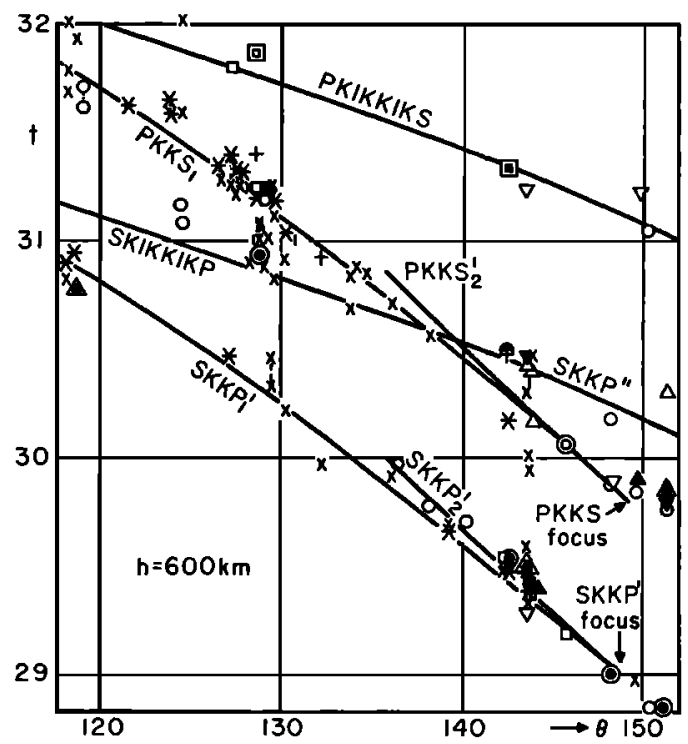

FIG. 5-Travel times $t$ of SKKP-PKKS group, details as in Fig. 1. 

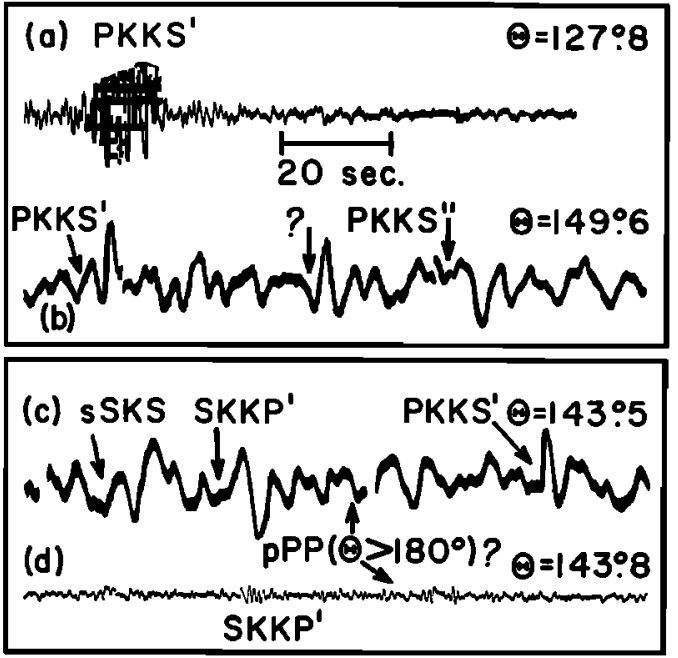

FIa. 6-Portions of records as in Fig. 2, showing waves in PKKS-SKKP group. (a) and (d) shortperiod Benioff vertical; (b) and (c) Wilson-Lamison NS; (a) Woody, $127.8^{\circ}$; (b) Columbia, $149.6^{\circ}$; (c) Fayetteville, $143.5^{\circ}$; (d) Dallas, $143.8^{\circ}$.

A number of travel-time curves of other waves (for example, sPS, SPP, PS, PSKS, SP, SKSP) intersect those of the group, but the relatively small periods of the waves under consideration and the fact that the travel times of the other waves increase with increasing distance, thus contrasting with those of the waves considered here, aids a definite identification of the waves in most instances. For most of the phases of the SKKP-PKKS group there are enough definite observations (Fig. 5) to establish that the observed and the calculated travel times agree within the limits of error and that the same is true for the distances of the focal points. Again, there are no observed waves along the trend of the curves at distances over about $2^{\circ}$ beyond the calculated focal points; that is, there is no indication of appreciable diffraction.

The fact that PKKS is frequently clear not only on the horizontal but also on the vertical components, in spite of the relatively steep arrival of the waves at the earth's surface, is explained by the theoretical ratio of the vertical ground motion to the amplitudes of the incident SV waves. This increases rather rapidly with increasing angle of incidence of SV and reaches about 0.7 for SV waves which have an angle of incidence at the surface of slightly less than $20^{\circ}$ [Gutenberg, 1944, p. 99].

SKKKP and PKKKS theoretically have a focal point at a distance of about $290^{\circ}\left(70^{\circ}\right)$ with a travel time of $381 / 4$ min for shocks originating at the surface. The factor $F$ for the amplitude reduction at the refractions and reflections at the core boundary should be noticeably smaller than 0.01 , so that it is unlikely that these phases are observed.

The wave periods-PKKP "has a short period" [Gutenberg and Richter, 1934, p. 116]. "In PKKP, waves with periods of three seconds or more are extremely rare on all records" [Gutenberg, 1951, p. 380]. Usually, PKKP waves are immediately identified on records of short-period instruments on the basis of their short periods (Fig. 2) ; PKKP' is rarely found on records of long-period instruments. No definite reason for the short periods of $\mathrm{PKKP}^{\prime}$ nor of those of $\mathrm{P}^{\prime} \mathrm{P}^{\prime}$ has been given thus far. On the records of the shock of April 16, 1957, periods of all well-recorded waves which had been reflected at the inside of the core boundary have been measured. In some instances, periods of waves from other phases, superposed on the waves under consideration, may have been measured, so that the 'observed' periods are rather too large than too small.

It was found that for statistical purposes the periods observed in $\mathrm{PKKP}^{\prime}$ and $\mathrm{PKKP}^{\prime \prime}$ may be combined, though those of PKKP" are on the average slightly longer than those of PKKP'. Similarly, it was found that, within the limits of error, periods of PKKS', PKKS", SKKP', and SKKP' may be combined, and also those of SKKS with those of SKKKS and SKKKS*. The respective period frequencies are given in the first three columns of Table 1, followed by those found for PP. The following columns are based on results of previous research. Where data from shocks at various focal depths are combined ("all" in Table 1), most refer to shallow shocks, and the number of data from deeper shocks decreases with focal depth [see, for example, Gutenberg, 1958c, Table 3, p. 277]. Usually, in deep shocks the periods are slightly smaller than the corresponding periods in shallow shocks.

Gutenberg [1958c, p. 275] has concluded that "the smallest periods observed regularly 
TABLE 1-Frequencies (in per cent) of periods $T$ in seconds observed in records of various phases, $h=$ depth of focus in $\mathrm{km}, n=$ number of observations

\begin{tabular}{|c|c|c|c|c|c|c|c|c|c|c|c|c|c|}
\hline Phase & & $\begin{array}{l}\text { SKKP } \\
\text { PKKS }\end{array}$ & $\begin{array}{l}\text { SKKS } \\
\text { SKKKS }\end{array}$ & PP & PKKP & PKKP & $\mathrm{P}^{\prime} \mathrm{P}^{\prime}$ & $\begin{array}{l}\mathbf{P}^{\prime} \\
\mathbf{P}^{\prime \prime}\end{array}$ & $\begin{array}{l}\text { SKP } \\
\text { PKS }\end{array}$ & $\mathbf{P}$ & $\mathbf{S}$ & $\mathbf{P}$ & $\mathbf{S}$ \\
\hline$h$ & 600 & $\begin{array}{c}600 \\
(1957, A\end{array}$ & $\begin{array}{c}600 \\
\text { pril } 16)\end{array}$ & 600 & $<70$ & all & all & all & all & all & all & $>300$ & $>300$ \\
\hline
\end{tabular}

\begin{tabular}{|c|c|c|c|c|c|c|c|c|c|c|c|c|c|}
\hline \multicolumn{14}{|l|}{$T$} \\
\hline$\leq \overline{1}$ & 70 & 41 & 3 & 15 & 43 & 47 & 27 & 42 & 17 & 22 & 0 & 35 & 3 \\
\hline $1.1-3$ & 24 & 39 & 23 & 49 & 56 & 52 & 66 & 51 & 51 & 24 & 1 & 31 & 5 \\
\hline $3.1-6$ & 6 & 13 & 56 & 31 & 1 & 1 & 7 & 7 & 28 & 26 & 20 & 27 & 53 \\
\hline $6.1-10$ & 0 & 7 & 15 & 3 & 0 & 0 & 0 & 1 & 3 & 23 & 44 & 7 & 32 \\
\hline$>10$ & 0 & 0 & 3 & 2 & 0 & 0 & 0 & 0 & 1 & 5 & 35 & 0 & 8 \\
\hline$n$ & 33 & 101 & 117 & 61 & 96 & 109 & 154 & 183 & 90 & 368 & 410 & 45 & 38 \\
\hline
\end{tabular}

in $\mathrm{S}$ waves at distances between $20^{\circ}$ and $100^{\circ}$ are about 4 seconds, while periods in $P$ waves of 1 to 3 seconds are frequently reported for all these distances." Table 1 shows that the shortest periods are observed in longitudinal phases which have traveled through the core (PKKP and $\mathrm{P}^{\prime} \mathrm{P}^{\prime}$ ); slightly greater periods are found in those waves which have traversed the mantle once as longitudinal and once as transverse waves (SKP, PKS, PKKS, SKKP). SKKS and SKKKS have still longer periods, but, in contrast with $\mathrm{S}$, frequently show periods of between 1 and 3 seconds.

It is difficult to explain these results. It is possible but not likely that greater attenuation of long waves than of short waves in the core is the main cause; it rather seems that a relative increase in the number and amplitudes of waves having shorter periods plays the main role (compare SKKS with S.) The fact that there seems to be little difference between the waves which have traveled through portions of the inner core and those which have not entered the transition zone between the outer and the inner core, puts the source of the short waves in the outer core or in the core boundary. In the records of the shock $600 \mathrm{~km}$ deep that is under special investigation, about 24 per cent of SKKS, but 39 per cent of SKKKS waves which have passed a portion of the outer core three times, had periods of 1 to 3 seconds; if the corresponding waves beyond $180^{\circ}$ are included, the corresponding figures are 22 and 39 per cent respectively. However, the number of observations ( $n$ in Table 1) is too small to prove that the shortening of the periods of SKKS and SKKKS occurs in the outer core, although the figures in Table 1 strongly support this conclusion; neither the SKKS nor the SKKKS waves involved have entered the inner core.

\section{REFERENCES}

Dana, S. W., The partition of energy among seismic waves reflected and refracted at the earth's core, Bull. Seis. Soc. Am., 34, 189-197, 1944.

Gutenberg, B. Fnergy ratio of reflected and refracted seismic waves, Bull. Seis. Soc. Am., 34, 85-102, 1944.

Gutenberg, B., PKKP, P'P', and the earth's core, Trans. Am. Geophys. Union, 32, 373-390, 1951.

Gutenherg, B., Caustics produced by waves through the earth's core, Geophys, J., 1, 238248, 1958 .

Gutenber, B., Wave velocities in the earth's core, Bull. Seis. Soc, Am., 48, 301-314, 1958 b.

Gutenberg, B., Attenuation of seismic waves in the earth's mantle, Bull. Seis. Soc. Am., 48, 269282, $1958 c$.

Gutenberg, B., and C. F. Richtar, On seismic waves (First paper), Gerlands Beitr. Geophys., 43, 56-133, 1934.

Gutenbera, B., and C. F. Richter, Materials for the study of deep-focus earthquakes, Bull. Seis. Soc. Am., 26, 341-390, 1936.

Gutenber,, B., and C. F. Richter, On seismic waves (Fourth paper), Gerlands Beitr. Geophys., 54, 94-136, 1939.

JefFeys, H., AND K. E. Bullen, Seismological Tables, British Assoc. Advance. Sci., 48 pp., 1940.

Nelson, R. L., A study of the seismic waves SKS and SKKS, Bull. Seis. Soc. Am., 44, 39-55, 1954.

(Manuscript received June 13, 1959.) 